\title{
A multivariate interval approach for inverse uncertainty quantification with limited experimental data
}

\author{
Matthias Faes ${ }^{\mathrm{a}, \mathrm{f}, *}$, Matteo Broggi ${ }^{\mathrm{b}, \mathrm{f}}$, Edoardo Patellic, Yves Govers ${ }^{\mathrm{d}}$, John \\ Mottershead $^{\mathrm{c}}$, Michael Beer ${ }^{\mathrm{b}, \mathrm{c}, \mathrm{e}}$, David Moens ${ }^{\mathrm{a}}$ \\ ${ }^{a}$ KU Leuven, Department of Mechanical Engineering, Technology campus De Nayer, Jan \\ De Nayerlaan 5, St.-Katelijne-Waver, Belgium \\ ${ }^{b}$ Leibniz Universität Hannover, Institute for Risk and Reliability, Callinstrasse 34, \\ Hannover, Germany \\ ${ }^{c}$ University of Liverpool, Institute for Risk and Uncertainty, Peach Street, L69 7ZF \\ Liverpool, United Kingdom \\ ${ }^{d}$ German Aerospace Center (DLR), Institute of Aeroelasticity, Bunsenstr. 10, Göttingen, \\ Germany \\ e Tongji University, International Joint Research Center for Engineering Reliability and \\ Stochastic Mechanics, Shanghai 200092, China \\ ${ }^{f}$ Both authors contributed equally to this paper.
}

\begin{abstract}
This paper introduces an improved version of a novel inverse approach for the quantification of multivariate interval uncertainty for high dimensional models under scarce data availability. Furthermore, a conceptual and practical comparison of the method with the well-established probabilistic framework of Bayesian model updating via Transitional Markov Chain Monte Carlo is presented in the context of the DLR-AIRMOD test structure. First, it is shown that the proposed improvements of the inverse method alleviate the curse of dimensionality of the method with a factor up to $10^{5}$. Furthermore, the comparison with the Bayesian results revealed that the selection of the most appropriate method depends largely on the desired information and availability of data. In case large amounts of data are available, and/or the analyst desires full (joint)-probabilistic descriptors of the model parameter uncertainty, the Bayesian method is shown to be the most performing. On the other hand however, when such descriptors are not needed (e.g., for worst-case analysis), and only scarce data are available,
\end{abstract}

\footnotetext{
* Corresponding author

Email address: matthias.faes@kuleuven.be (Matthias Faes)
}

Preprint submitted to Mechanical Systems and Signal Processing 
the interval method is shown to deliver more objective and robust bounds on the uncertain parameters. Finally, also suggestions to aid the analyst in selecting the most appropriate method for inverse uncertainty quantification are given.

Keywords: Multivariate interval uncertainty, Uncertainty Quantification, DLR-AIRMOD, Bayesian model updating, Limited data

\section{Introduction}

In general engineering practice, the knowledge on a structure is usually incomplete, be it due to inherent variable model parameters or a lack of knowledge on the true parameter values [1, 2]). Hence, representing these model param5 eters as deterministic quantities might prove to be inadequate when a reliable and economic design is pursued, as a large degree of conservatism is needed to prevent premature failure and corresponding maintenance or insurance costs.

This over-conservatism not only impairs the economic cost of producing the component; it also leads to unnecessary weight increase, which is impermissible in high-performance sectors such as machinery design, aerospace or automotive. In the last few decades, highly advanced techniques including probabilistic [3], possibilistic [4 or imprecise probabilistic methods [5] have been introduced to include non-determinism efficiently in these design models.

In order for these tools to deliver a realistic quantification of the nondeterminism in the responses of the design model, the description of the nondeterministic parameters of the model should be made objectively and accurately. Since not all parameters (such as e.g. connection stiffness values or heterogeneous material properties) are trivial to measure directly, inverse uncertainty quantification (UQ) techniques have been introduced. Following inverse UQ, the responses of the structure are measured and used to infer knowledge on the non-determinism in the model parameters. As concerns inverse UQ in a probabilistic sense, the class of Bayesian methods is considered the standard approach [6], even for random fields [7. However, in the context of limited, insufficient, vague or ambiguous data, the prior estimation of the joint proba- 
bility density function of the non-deterministic parameter values is subjective. Moreover this estimate influences the quantified result to a large extent when insufficient independent measurement data are available.

Inverse UQ methods for the identification and quantification of multivariate interval uncertainty usually minimise a squared $\mathcal{L}_{2}$-norm over the difference

30 between the interval boundaries of respectively a measurement data set and the prediction of the FE model [8, 9]. Application of most of these techniques is prone to ill-conditioning and non-uniqueness when no special care is taken in the definition of the identification problem [10]. Also alternative approaches using Kriging predictor models were introduced recently [11] and compared to 35 stochastic model updating [12].

Recently, a novel methodology for the identification of multivariate interval uncertainty was introduced by some of the authors in [13], with an extension to interval fields in [14] and [15]. This method is based on the convex hull concept for the representation of dependent uncertain output quantities of an

40 interval FE model, and iteratively minimises the discrepancy between the convex hull of these uncertain output quantities with the convex hull over a set of replicated measurement data. However, since the computation of a convex hull follows an exponential time complexity with its dimension, the dimension of these convex hulls should be reduced as to allow for applying this method to

45 large-scale problems. Dimension reduction is a topic that is quickly emerging in the fields of big data and machine learning, where datasets are often too highdimensional to be handled directly. In this context, a broad range of techniques based on for instance covariance matrix decompositions [16], manifold learning approaches [17, or active subspace methods [18] have been introduced in recent ${ }_{50}$ years. In the context of the inverse quantification of multi-dimensional interval uncertainty, the application of such dimension reduction methods is an underexplored domain.

Finally, whereas the literature on comparing forward UQ in a probabilistic and non-probabilistic context is abundant (see e.g., [19] or [20] for a more re55 cent treatment), such a practical comparison for inverse approaches is severely 
lacking in literature. The objective of this paper is therefore twofold. First, an improved version of a recently proposed interval method [13, 14] is presented in the sense that by reducing the dimension of the corresponding convex hulls, more challenging problems can be tackled. In addition, an objective comparison of Bayesian uncertainty quantification methods [21], which are most commonly applied in a probabilistic context is provided and suggestions for choosing the most appropriate technique based on the data are made.

The paper is structured as follows. Section 2 introduces the extensions to the novel method for the identification and quantification of multivariate interval uncertainty. It is illustrated that the exponential time complexity of computing the objective function is relaxed by projecting the convex hull onto lower-dimensional subspaces of an orthogonal basis with a dimension equal to the effective dimension of the convex hulls. Section 3 presents the reader with the concept of Bayesian uncertainty quantification. Both techniques are critically compared and a conceptual comparison is given in section 4 . Section 5 presents a case study comparing the applicability of both methods to the well-known DLR-AIRMOD 22 case. Specifically, it is studied how both methods perform in terms of obtained information, computational cost and accuracy, depending on the size of the dataset. Finally, section 6 lists the conclusions of this work.

\section{2. Multivariate interval quantification}

This section introduces the interval finite element method and the method used for the identification and quantification of multivariate interval uncertainty based on indirect measurement data. In the following, a model parameter $\theta$ having interval uncertainty is denoted $\theta^{I}$. Vectors are expressed as lower-case boldface characters $\boldsymbol{\theta}$. Interval parameters are either represented using the bounds of the interval $\theta^{I}=[\underline{\theta} ; \bar{\theta}]$ or the centre point $\hat{\theta}=\frac{\underline{\theta}+\bar{\theta}}{2}$ and the interval radius $r_{x}=\frac{\bar{\theta}-\underline{\theta}}{2}$. 


\subsection{The interval finite element method}

Let $\mathcal{M}$ be a deterministic Finite Element model that is used to solve a (set of) differential equations for $\boldsymbol{z}^{\boldsymbol{m}} \in \mathbb{R}^{d}$ through the vector valued function operator $g:$

$$
\mathcal{M}(\boldsymbol{\theta}): \boldsymbol{z}^{\boldsymbol{m}}=g(\boldsymbol{\theta}), \quad g: \mathbb{R}^{k} \mapsto \mathbb{R}^{d}
$$

with $\boldsymbol{\theta} \in \mathcal{F} \subset \mathbb{R}^{k}$ the vector of model parameters and $\mathcal{F}$ the sub-domain of feasible parameters (e.g., non-negative contact stiffness).

The uncertainty that is attributed to $\boldsymbol{\theta}$ is modeled as an interval vector $\boldsymbol{\theta}^{I} \in \mathcal{F}^{I} \subset \mathbb{I}^{k}$, with $\mathbb{R}^{k}$ the space of $k$-dimensional interval vectors. Note that due to the orthogonality of all $\theta_{i}, i=1, \ldots, k$, all model parameters are considered independent by definition. Therefore, $\boldsymbol{\theta}^{I}$ can also be represented as a $k$-dimensional hypercube.

The interval FE method generally aims at finding a solution set $\tilde{\boldsymbol{z}}$ containing the extreme realizations of $z^{m}$ given the hyper-cubic parameter uncertainty. In general, $\tilde{z}$ is not hyper-cubic, but spans a non-convex region in $\mathbb{R}^{d}$, since $\mathcal{M}$ provides coupling between $z_{i}^{m}, i=1, \ldots, d$. Therefore, it is commonly approximated by an uncertain realization set $\tilde{\boldsymbol{z}}^{m}$, which is obtained by propagating $q$ well selected deterministic realizations $\boldsymbol{z}_{j}^{m}$ of the hyper-cubic uncertain input parameters $\boldsymbol{\theta}^{I}$ :

$$
\tilde{\boldsymbol{z}}^{m}=\left\{\boldsymbol{z}_{j}^{m} \mid \boldsymbol{z}_{j}^{m}=\mathcal{M}\left(\boldsymbol{\theta}_{i}\right) ; \boldsymbol{\theta}_{i} \in \boldsymbol{\theta}^{I} ; i=1, \ldots, q\right\}
$$

In general, $\tilde{\boldsymbol{z}}^{m} \approx \tilde{\boldsymbol{z}}$ when $q$ is taken sufficiently large, or sampled intelligently (e.g., following the Cauchy deviates method [23]). When $\mathcal{M}$ is a strictly monotonic FE model, the Transformation Method [24] provides an exact mapping from $\boldsymbol{\theta}^{I}$ to $\tilde{\boldsymbol{z}}$, albeit needing $2^{k}$ deterministic model evaluations for the solution 95 of a single interval problem.

A mathematical handle to the boundaries of $\tilde{\boldsymbol{z}}^{m}$ is provided by the convex hull $\mathcal{C}^{m}$ of the realization set $\tilde{\boldsymbol{z}}^{m} \cdot \mathcal{C}^{m}$ can be considered as a set of vertices 
bounding the uncertain realization set:

$$
\mathcal{C}^{m}=\left\{\sum_{j=1}^{q} \beta_{j} \boldsymbol{z}_{j}^{m} \mid\left(\forall j: \beta_{j} \geq 0\right) \wedge \sum_{j=1}^{q} \beta_{j}=1 ; \boldsymbol{z}_{j}^{m} \in \tilde{\boldsymbol{z}}^{m}\right\}
$$

with $\beta$ a vector of weighting factors, such that all elements $\beta_{j}$ are non-negative and sum to one. Such convex hull has also a multi-dimensional volume $\mathcal{V}_{m}$. Alternatively, $\mathcal{C}^{m}$ can also be represented as a set of $h_{m} d$-dimensional linear inequalities that describe the boundaries of $\mathcal{C}^{m}$ :

$$
\mathcal{C}^{m} \equiv\left[f_{1}, \ldots, f_{h_{m}}\right]^{T} \equiv \mathbf{A}_{m}\left(\boldsymbol{z}^{m}\right)^{T}-\mathbf{b}_{m} \geq 0
$$

with $\mathbf{A}_{m} \in \mathbb{R}^{h_{m} \times d}, \mathbf{b}_{m} \in \mathbb{R}^{h_{m}}$ and $h_{m}$ the number of half-spaces $f_{i}, i=$ $1, \ldots, h_{m}$. An illustration of such convex hull over 12 arbitrary realisations $\boldsymbol{z}_{j}^{m}$ of two responses $z_{1}$ and $z_{2}$ is given in figure 1 .

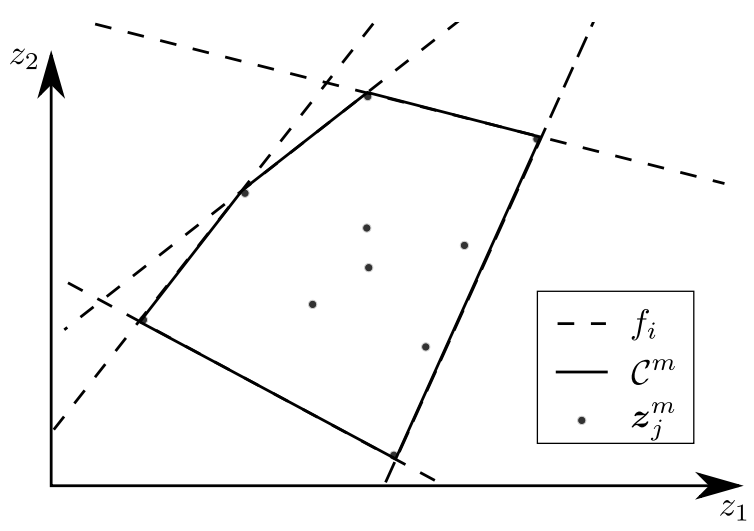

Figure 1: Convex hull and bounding halfspaces over 12 realisations $\boldsymbol{z}_{j}^{m}$ of two arbitrary responses $z_{1}$ and $z_{2}$ using five half-spaces $f_{i}$.

\subsection{Multivariate interval quantification}

This section presents an improved version of the method presented in [13, 14. Specifically, the reduction of the computational expense is extended as to accommodate high-dimensional FE models. First the basis method is introduced for the readers convenience.

As a basis for the inverse quantification of multivariate interval uncertainty, experimental data $\mathcal{D}$ of the model responses $\boldsymbol{z}$ are obtained by experimentally 
testing $t$ times a physical replica of the numerical model. These measurements are then used to construct a measurement set $\tilde{\boldsymbol{z}}^{e}$, and the non-determinism that is present in these replicated measurements is bounded by its convex hull $\mathcal{C}^{e}$ and corresponding $d$-dimensional volume $\mathcal{V}_{e}$ in analogy to eq. (3). These computations are performed using the QuickHull algorithm [25].

A specific issue arises when $d$ becomes large (say $d>10$ ). In that case, computation of a single convex hull might take prohibitively long since the worst-case time complexity QuickHull is:

$$
\mathcal{O}\left(\left\lfloor v_{c}^{\frac{d}{2}}\right\rfloor /\left\lfloor\frac{d}{2}\right\rfloor !\right)
$$

with $v_{c}$ the number of vertices of $\mathcal{C}^{m}[25$.

To accommodate this, the dimension of the vector space $\mathbb{R}^{d}$ in which these convex hulls are computed, is reduced using the method presented in [14]. This method is briefly recalled in the following paragraphs. Specifically, an orthogonal basis $\mathcal{B}$ is constructed in $\mathbb{R}^{d_{r}}$, with $d_{r}<<d$. This basis is defined as:

$$
\mathcal{B}=\operatorname{span}\left\{\phi_{e, d-d_{r}}, \phi_{e, d-d_{r}+1}, \ldots \phi_{e, d}\right\}
$$

with $d_{r}$ chosen such that all non-zero dimensions are included in $\mathcal{B}$, and $\phi_{e}$ the eigenvectors corresponding to the $d_{r}$ largest eigenvalues of the covariance matrix $\boldsymbol{\Xi}_{e}$ of the measurement data set $\tilde{\boldsymbol{z}}^{e}$. These eigenvectors are obtained by following singular value decomposition:

$$
\boldsymbol{\Xi}_{\boldsymbol{e}}=\boldsymbol{\Phi}_{e} \boldsymbol{\Lambda}_{e} \boldsymbol{\Phi}_{e}^{T}
$$

with $\boldsymbol{\Lambda}_{e} \in \mathbb{R}^{d \times d}$ the diagonal matrix of the ordered eigenvalues $\lambda_{1} \leq \lambda_{2} \leq$ $\cdots \leq \lambda_{d}$ of $\boldsymbol{\Xi}_{e}$, and $\boldsymbol{\Phi}_{e} \in \mathbb{R}^{d \times d}$ a matrix containing the orthogonal eigenvectors $\phi_{e, j} \in \mathbb{R}^{d}, j=1, \ldots, d$. Standard Matlab routines are used to perform the decomposition, which make use of Blas and LAPACK libraries. Note that since $\Xi_{e}$ is symmetric and positive definite by definition, the result of eq. 77 is exactly the same as when performing an eigenvalue decomposition. The covariance 
matrix $\boldsymbol{\Xi}_{e}$ is specifically constructed as:

$$
\boldsymbol{\Xi}_{e}=\left[\begin{array}{cccc}
\operatorname{var}\left(\mathbf{z}_{e 1}^{t}\right) & \operatorname{cov}\left(\mathbf{z}_{e 1}^{t}, \mathbf{z}_{e 2}^{t}\right) & \ldots & \operatorname{cov}\left(\mathbf{z}_{e 1}^{t}, \mathbf{z}_{e d}^{t}\right) \\
\operatorname{cov}\left(\mathbf{z}_{e 2}^{t}, \mathbf{z}_{e 1}^{t}\right) & \operatorname{var}\left(\mathbf{z}_{e 2}^{t}\right) & \ldots & \operatorname{cov}\left(\mathbf{z}_{e 2}^{t}, \mathbf{z}_{e d}^{t}\right) \\
\vdots & & \ddots & \vdots \\
\operatorname{cov}\left(\mathbf{z}_{e d}^{t}, \mathbf{z}_{e 1}^{t}\right) & \operatorname{cov}\left(\mathbf{z}_{e 1}^{t}, \mathbf{z}_{e 2}^{t}\right) & \ldots & \operatorname{var}\left(\mathbf{z}_{e d}^{t}\right)
\end{array}\right]
$$

with $\mathbf{z}_{e j}^{t} \in \mathbb{R}^{t}, j=1, \ldots, d$ a vector containing all measured replica for a single response, and $t$ the number of replicated measurements. Finally, the number of needed eigenvectors $d_{r}$ is then selected as:

$$
\sum_{i=1}^{d_{r}} \frac{\lambda_{e, i}}{\operatorname{tr}\left(\boldsymbol{\Xi}_{e}\right)} \geq 1-\epsilon
$$
on minimising the discrepancy between these convex hulls, all computations for the quantification are performed in $\mathcal{B}$.

However, when realistic numerical models containing thousands of degree'sof-freedom and/or measurement sets consisting of numerous responses are considered, the effective dimension $d_{r}$ still might be prohibitively large (i.e., $d_{r}>$ 10). Therefore, as an extension to this previously presented dimension reduction method, it is proposed to further reduce the dimension of the vector space $\mathbb{R}^{d_{r}}$ in which both convex hulls are defined. Specifically, both $\tilde{\boldsymbol{z}}_{m}^{r}$ and $\tilde{\boldsymbol{z}}_{e}^{r}$ are fur- 
ther projected onto $d_{r}^{+}$-dimensional subspaces, defined by a lower-dimensional orthogonal basis $\mathcal{B}_{i}^{+} \subset \mathcal{B}, i=1, \ldots,\left(\begin{array}{c}d_{r} \\ d_{r}^{+}\end{array}\right)$, constructed as a subset of $\mathcal{B}$, with $d_{r}^{+}<<d_{r}$ and $\left(\begin{array}{l}d_{r} \\ d_{r}^{+}\end{array}\right)$the binomial coefficient. Specifically, the $i^{t h}$ orthogonal subspace basis $\mathcal{B}_{i}^{+}$is defined as:

$$
\mathcal{B}_{i}^{+}=\operatorname{span}\left\{\phi_{m, \mathcal{I}_{i}(1)}, \phi_{m \mathcal{I}_{i}(2)}, \ldots \phi_{m, \mathcal{I}_{i}\left(d_{r}^{+}\right)}\right\}
$$

with $\mathcal{I}_{i}$ an index set containing the $d_{r}^{+}$indices for the $i^{t h}, i=1, \ldots,\left(\begin{array}{l}d_{r} \\ d_{r}^{+}\end{array}\right)$subspace of $\mathcal{B}$. Then, the reduced sets $\tilde{\boldsymbol{z}}_{e}^{r}$ and $\tilde{\boldsymbol{z}}_{m}^{r}$ are projected on these subspaces, i.e. $\tilde{\boldsymbol{z}}_{\mathcal{B}_{i}^{+}}^{e}$ and $\tilde{\boldsymbol{z}}_{\mathcal{B}_{i}^{+}}^{m}$, and their respective convex hulls $\mathcal{C}_{\mathcal{B}_{i}^{+}}^{e}$ and $\mathcal{C}_{\mathcal{B}_{i}^{+}}^{m}$ with corresponding $d_{r}^{+}$-dimensional volumes $\mathcal{V}_{e}$ and $\mathcal{V}_{m}$ are computed using the functionality of the QHULL library. By applying this reduction scheme, the time complexity of the computation of the convex hull, which is originally defined as in eq. (5), becomes:

$$
\mathcal{O}\left(\left\lfloor v_{c}^{\frac{d_{r}^{+}}{2}}\right\rfloor /\left\lfloor\frac{d_{r}^{+}}{2}\right\rfloor ! \times\left(\begin{array}{c}
d_{r} \\
d_{r}^{+}
\end{array}\right)\right)
$$

Therefore, as long as $d_{r}^{+}$is a very small number, the computational cost of calculating $\left(\begin{array}{l}d_{r} \\ d_{r}^{+}\end{array}\right) d_{r}^{+}$dimensional convex hulls is considerably smaller than computing a single $d_{r}$ dimensional convex hull. This projection however only retains the $d_{r}^{+}-1$ order interactions between model responses. Herein, $d_{r}^{+}=1$ is the limit case where only the hyper-cubic approximation of $\tilde{\boldsymbol{z}}_{m}^{r}$ and $\tilde{\boldsymbol{z}}_{e}^{r}$ is retained. This however does not limit the accuracy of the method as long as $d_{r}^{+} \geq 2$, since by considering only the convex hulls all higher-order interactions between model responses are already linearised. Therefore, no further approximation of the dependence structure is made.

Finally, the multivariate interval uncertainty in $\boldsymbol{\theta}^{I}$ is obtained by minimizing following objective function:

$$
\delta\left(\boldsymbol{\theta}^{I}\right)=\sum_{i=1}^{\substack{d_{r} \\ d_{r}^{+}}}\left(\Delta V_{m, i}^{2}+w_{o} \Delta V_{o, i}^{2}+\Delta c_{i}^{2}\right)
$$


with:

$$
\begin{aligned}
& \Delta V_{m, i}=1-\frac{\mathcal{V}_{m, i}\left(\boldsymbol{\theta}^{I}\right)}{\mathcal{V}_{e, i}} \\
& \Delta V_{o, i}=1-\frac{\mathcal{V}_{o, i}\left(\boldsymbol{\theta}^{I}\right)}{\mathcal{V}_{e, i}} \\
& \Delta c_{i}=\left\|\mathbf{c}_{e, i}-\mathbf{c}_{m, i}\left(\boldsymbol{\theta}^{I}\right)\right\|_{2}
\end{aligned}
$$

where $\mathcal{V}_{m, i}$ and $\mathcal{V}_{e, i}$ are the $d_{r}^{+}$-dimensional volumes of respectively $\mathcal{C}_{\mathcal{B}_{i}^{+}}^{m}$ and $\mathcal{C}_{\mathcal{B}_{i}^{+}}^{e}, \mathcal{V}_{o, i}$ is the volume of the overlap of both convex hulls, and $\mathbf{c}_{e, i}$ and $\mathbf{c}_{m, i}$ simplicity, the subscript $\mathcal{B}_{i}^{+}$is simplified to $i$.

The advantage of this methodology for reducing the dimension over selecting a smaller value for $d_{r}$, is that more information on the dependence and nondeterminism that is present in the model quantities is retained in the analysis. imise the difference between the multidimensional volumes between the convex hull of the measurement data set and the convex hull resulting from the interval FE analysis, as to maximise the overlap between these convex hulls. As the set of measurement data generally does not contain the same extreme vertices as contained in the interval model, the volumes cannot be matched exactly. Therefore, a trade-off is found by the optimiser, where the volume of the convex hulls is matched at the price of losing some overlap. Increasing the factor $w_{o}$ 
alleviates this problem, however at the cost of increasing the over-conservatism of the prediction (see e.g., [14]) and making the optimisation problem harder to solve due to the barrier-function like behaviour of the term describing the overlap.

\section{Bayesian model updating}

The use of Bayesian methods for uncertainty quantification is largely founded on the the pioneering work of Beck and Katafygiotis [6, 21] in the late 1990s. Following the Bayesian interpretation of probability, the probabilistic nature of an uncertain parameter is interpreted as the degree to which it is believed that each possible value of this parameter is consistent with the available information (e.g., the response of a high-fidelity model or measurement data). Following Bayes' rule, this degree of belief is adjusted using independent information. As such, the Bayesian methods translate this prior knowledge on the uncertainty corresponding to the parameter values to an updated posterior knowledge, based on experimental data.

As a first step in the uncertainty characterisation, a prior probability distribution $p(\Theta \mid \mathcal{M})$, conditioned upon a chosen mathematical model $\mathcal{M}$, is assigned to a set of uncertain parameters $\boldsymbol{\Theta}$. These distributions represent prior information on the uncertain parameters, stemming from e.g., expert opinions, lab-scale specimen testing, previous quantification of the uncertainty, etc. Then, experimental data $\mathcal{D}$ are used to update this prior knowledge by means of Bayes' theorem, yielding the posterior distribution $p(\boldsymbol{\Theta} \mid \mathcal{D}, \mathcal{M})$ :

$$
p(\boldsymbol{\Theta} \mid \mathcal{D}, \mathcal{M})=\frac{p(\mathcal{D} \mid \Theta, \mathcal{M}) p(\boldsymbol{\Theta} \mid \mathcal{M})}{p(\mathcal{D} \mid \mathcal{M})}
$$

where $p(\mathcal{D} \mid \Theta, \mathcal{M})$ is the likelihood of obtaining the data $\mathcal{D}$, given the value of the uncertain parameters $\Theta$ and a specific model $\mathcal{M}$. The denominator of 180 eq. 14, also commonly referred to as evidence, ensures that the posterior distribution $p(\boldsymbol{\Theta} \mid \mathcal{D}, \mathcal{M})$ integrates to one.

In the context of structural dynamics, the data $\mathcal{D}$ usually consists of the residuals between experimental measurements and predictions of the model $\mathcal{M}$ 
as:

$$
\epsilon_{i}=z_{i}^{e}-z_{i}^{m}(\boldsymbol{\Theta}), \quad i=1, \ldots, n
$$

where $z_{i}^{e}=\omega_{i}^{2}$ is the square of the $i$-th measured response, $z_{i}^{m}(\boldsymbol{\Theta})=\lambda_{i}(\boldsymbol{\Theta})$ is the $i$-th predicted response of a finite element model and $n$ the number of considered responses. In the specific context of parameter uncertainty quantification using experimental modal analysis data, $z_{i}^{e}$ and $z_{i}^{m}$ correspond to measured and predicted eigenfrequencies of $\mathcal{M}$.

In practice, the likelihood function is often chosen to be a zero-mean multivariate normal distribution [29]:

$$
p(\mathcal{D} \mid \boldsymbol{\Theta}, \mathcal{M})=\prod_{i=1}^{N} \frac{1}{(2 \pi)^{n / 2}|\Sigma|^{1 / 2}} \exp \left(-\frac{1}{2} \epsilon_{i}^{T}(\boldsymbol{\Theta}) \boldsymbol{\Sigma}^{-1} \epsilon_{i}(\boldsymbol{\Theta})\right)
$$

where $N$ denotes the number of data points in $\mathcal{D}$. The solution to eq. $(14)$ is commonly approximated by sampling from a Markov Chain that is ergodic and stationary with respect to $p(\mathcal{D} \mid \Theta, \mathcal{M})$. In this paper, the transitional Markov Chain Monte Carlo (TMCMC) method, as introduced by Ching and Chen [30], is applied for the computation of the posterior distribution.

\section{Conceptual comparison of Bayesian and interval methods for in- verse UQ}

The methods presented in section 2 and 3 can both be used for inverse uncertainty quantification, but the underlying philosophy differs greatly. Similarly to forward uncertainty quantification, probabilistic and interval approaches are complementary, and the most appropriate method given an inverse UQ problem is highly case dependent. Important contrasts, next to the obvious philosophical differences, include the availability of objective and informative prior information, and even more so, measurement data of the model responses and the desired information content on the uncertain model parameters. This section aims at guiding the analyst through the selection of the most appropriate method. 
Interval methods are based on the idea that the possible values of the undice that favours the numbers 4 and 5 . The analyst, not knowing about the bias on the dice, has a prior assumption that the probability of rolling one of each 
number in the set $\{1,2,3,4,5,6\}$ is equal. When the analyst starts rolling the be obtained from the analysis. Finally, as long as the measurement data are obtained in an optimized way (i.e., such that they completely capture the needed 
scatter in the responses that results from the uncertainty that is studied), interval methods provide sufficient information for worst-case analysis.

\subsection{Bayesian or interval?}

In case large data sets are available or the analyst has need for quantifying the relative likelihood of several parameter values being realised, including their (joint)-plausibility, correlation and multi-modal descriptors, Bayesian methods have the upper hand over interval approaches, but the analyst should ensure that sufficient informative data are available as discussed in section 4.1. On the other hand, when data are vague or scarce, interval methods are expected to provide a more objective and accurate quantification of the uncertainty, as less a priori assumptions on the underlying likelihood structure are needed. This however is achieved at the cost that only worst-case information is delivered to the analyst. This information is summarised in figure 2.

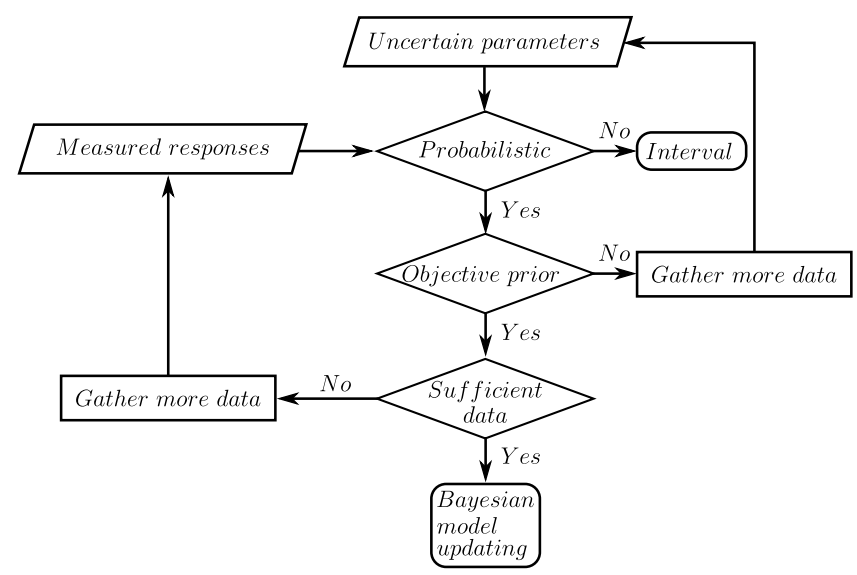

Figure 2: Selection flowchart to aid the analyst choosing the most appropriate method

\section{Application: the DLR-AIRMOD test structure}

\subsection{Introduction}

The first goal of this case study is to validate the novel reduction method that is presented in the context of the multivariate interval quantification. Secondly, 
it also aims at providing an in-depth discussion concerning the applicability of Bayesian and interval methods on a realistic dataset. The DLR-AIRMOD structure, with corresponding dataset (see [22]) is selected due to its challenging nature and the elaborate literature on the subject. Both presented techniques are applied to this case, and the results are compared in terms of obtained information and accuracy. The inverse UQ is first performed using the complete experimental data set containing 86 measured eigenfrequencies. Then, to illustrate the performance of both methods under scarce data, the inverse UQ is also performed using only 5 measured replica that were randomly selected from the full dataset. Note that, since both methods are conceptually very different, a quantitative comparison of the accuracy of the obtained results is not possible. Therefore, the comparison is made qualitatively in terms of conservatism with respect to the measurement data set.

Finally, since both methods require a large number of function evaluations, Artificial Neural Networks are used as surrogate models. Specifically a set of 2-layer (18:16:14:1) Neural Networks that map each vector of uncertain model parameter to one eigenfrequency of the FE model is constructed using state-ofthe art ANN tools and ensuring proper performance (see also 33] or 34 for a discussion of the training and validation).

\subsection{Model introduction}

The DLR AIRMOD structure, as shown in figure 3, is a scaled replica of the GARTEUR SM-AG19 benchmark airplane model 22. The physical AIRMOD structure is constructed from six aluminum beams that are connected by five bolted joints and weighs approximatly $40 \mathrm{~kg}$ to represent the fuselage, wings, winglets, vertical tail plate (VTP) and horizontal tail plate (HTP). It has a wing span of $2.0 \mathrm{~m}$, the fuselage length is $1.5 \mathrm{~m}$ and the height is $0.46 \mathrm{~m}$. The complete FE model, constructed in NX Nastran, consists of 1440 CHEXA, 6 CPENTA and 561 CELAS1, 55 CMASS1, 18 CONM2 and 3 CROD elements, and is constructed after 22 .

A set of 18 parameters including support and joint stiffness values, as well as 
mass parameters are selected for the identification (see table 1), in correspondence with literature on the subject [12. The locations of these parameters are indicated in figure 3 .

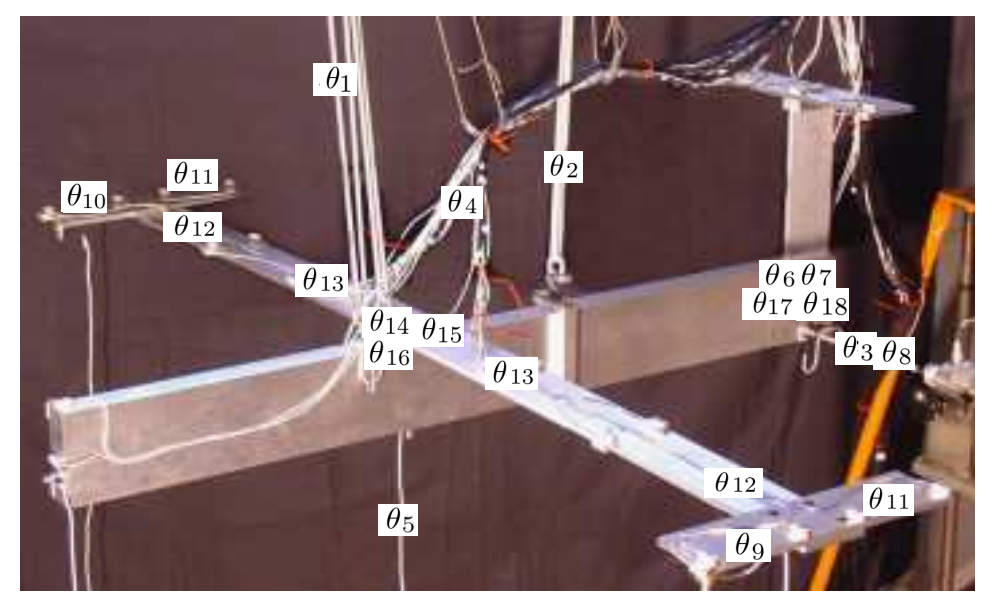

Figure 3: Illustration of the AIRMOD test structure (adapted after [22])

Table 2 lists the eigenmodes and eigenfrequencies that are obtained by solving the deterministic model (denoted as $f_{F E, \text { init }}$ ). From this table, it can be noted that the model indeed exhibits some challenges for uncertainty quantification procedures such as asymmetric modal behaviour and closely spaced modes. To prevent that perturbations in the model parameters lead to eigenmode veering or cross-over, MAC-based mode tracking with respect to the reference solu320 tion is applied in each stage of the quantification. From this set, the $1^{\text {st }}-8^{\text {th }}$, $10^{t h}-12^{t h}, 14^{t h}, 19^{t h}$ and $20^{t h}$ mode are selected for the identification. These 14 modes are selected to be consistent with literature on the subject [12].

It can be shown that the eigenfrequencies predicted by a linear numerical model are a strict monotonous function of the uncertain model parameters [35. In this case, the reduced transformation method 24] yields the exact bounds on the eigenfrequencies. However, the number of necessary function evaluations for the propagation scales exponentially with the number of uncertain model parameters. For the multivariate interval quantification method, it is therefore assumed that the masses at both wing-tips (i.e. $\theta_{9}$ and $\theta_{10}$ ) and the stiffness 
Table 1: Parameters that are used in the identification

\begin{tabular}{lllll}
\hline & Type & Description & Orientation & Deterministic value \\
\hline$\theta_{1}$ & Stiffness & Support stiffness & $y$ & $1.80 \cdot 10^{03} \mathrm{~N} / \mathrm{m}$ \\
$\theta_{2}$ & Stiffness & Support stiffness & $y$ & $7.50 \cdot 10^{03} \mathrm{~N} / \mathrm{m}$ \\
$\theta_{3}$ & Stiffness & Cables & $y$ & $1.30 \cdot 10^{02} \mathrm{~N} / \mathrm{m}$ \\
$\theta_{4}$ & Stiffness & Cables & $y$ & $7.00 \cdot 10^{01} \mathrm{~N} / \mathrm{m}$ \\
$\theta_{5}$ & Stiffness & Cables & $y$ & $7.00 \cdot 10^{01} \mathrm{~N} / \mathrm{m}$ \\
$\theta_{6}$ & Stiffness & Joint stiffness & $x, y$ & $1.00 \cdot 10^{07} \mathrm{~N} / \mathrm{m}$ \\
$\theta_{7}$ & Stiffness & Joint stiffness & $z$ & $1.00 \cdot 10^{09} \mathrm{~N} / \mathrm{m}$ \\
$\theta_{8}$ & Mass & Cables & $/$ & $2.00 \cdot 10^{-01} \mathrm{~kg}$ \\
$\theta_{9}$ & Mass & Screws & $/$ & $1.86 \cdot 10^{-01} \mathrm{~kg}$ \\
$\theta_{10}$ & Mass & Screws & $/$ & $1.86 \cdot 10^{-01} \mathrm{~kg}$ \\
$\theta_{11}$ & Mass & Cables & $/$ & $1.50 \cdot 10^{-02} \mathrm{~kg}$ \\
$\theta_{12}$ & Mass & Cables & $/$ & $1.50 \cdot 10^{-02} \mathrm{~kg}$ \\
$\theta_{13}$ & Mass & Cables & $/$ & $1.50 \cdot 10^{-02} \mathrm{~kg}$ \\
$\theta_{14}$ & Stiffness & Joint stiffness & $\mathrm{x}$ & $2.00 \cdot 10^{07} \mathrm{~N} / \mathrm{m}$ \\
$\theta_{15}$ & Stiffness & Joint stiffness & $\mathrm{y}$ & $2.00 \cdot 10^{07} \mathrm{~N} / \mathrm{m}$ \\
$\theta_{16}$ & Stiffness & Joint stiffness & $\mathrm{z}$ & $7.00 \cdot 10^{06} \mathrm{~N} / \mathrm{m}$ \\
$\theta_{17}$ & Stiffness & Joint stiffness & $\mathrm{x}$ & $5.00 \cdot 10^{07} \mathrm{~N} / \mathrm{m}$ \\
$\theta_{18}$ & Stiffness & Joint stiffness & $\mathrm{y}$ & $1.00 \cdot 10^{07} \mathrm{~N} / \mathrm{m}$ \\
\hline
\end{tabular}

330 $\left.\theta_{5}\right)$ are completely dependent, reducing the number of uncertain parameters to 16. Hence, the number of necessary function evaluations for a single interval computation reduces from 262144 to 65536 which makes it computationally more tractable. The validity of this assumption will be estimated based on 335

introduced by the cables at the top and the bottom of the structure $\left(\theta_{4}\right.$ and the obtained results. This assumption is not made for the Bayesian method, as the Monte Carlo sampling that underlies the applied TMCMC approach is dimension-independent. 


\subsection{Measurement data set}

A measurement data set containing 86 measurements of the 30 eigenmodes

340

For a complete explanation of the experimental campaign that was followed to construct this dataset, the reader is referred to [22]. The measured eigenfrequencies and their variability are shown in Table 2. In this table $\mu_{f}$ and $\sigma_{f}$ denote respectively the mean and standard deviation of the measurement data.

345 To assess the robustness of both methods against the size of measurement data, both the full measurement data set and a subset of 5 arbitrarily drawn replica were employed for the UQ of the 18 parameters.

\subsection{Multivariate interval quantification}

\subsubsection{Response set dimension reduction}

350

The number of considered model responses proves to be challenging in the application of the interval analysis due to the exponential time complexity of the applied QuickHull algorithm. The computation of a convex hull over all 14 pre-selected resonance frequencies, would therefore make the identification computationally intractable, as this computation has to be performed numerously during the quantification procedure. Therefore, this dimension is reduced following the method presented in section 2.2 In this context, application of equations 8 to 9 indicates that the effective dimensionality is equal to 13 when an approximation error $\epsilon$ below $0.1 \%$ is desired. This also can be seen in figure 4 which plots the reduction error $\epsilon$ as a function of the reduced dimension $d_{r}$ for the first $18 \lambda_{e, i}$.

Based on the singular value decomposition of the covariance matrix (see eq. (8)) of the pre-selected 14 eigenmodes, an orthogonal basis $\mathcal{B} \in \mathbb{R}^{13}$ is constructed according to eq. (6), and these 14 eigenmodes are projected onto this basis. This dataset is further projected onto lower-dimensional bases, in accordance with eq. 107. The computational gain of applying $d_{r}^{+}=2$ dimensional sub-bases of $\mathcal{B}$ is illustrated in figure 5 . This figure shows the wall-clock time that is needed to compute $1 d_{r}$-dimensional convex hull, as well as the time needed 
Table 2: eigenmodes and corresponding initial FE estimate of the corresponding eigenfrequency, as well as the statistics of the measured eigenfrequencies. The eigenmode naming convention is as follows. The letter before the dash - denotes the considered part, the letters after the dash the deformation mode. RB denotes the rigid body modes, $\mathrm{W}$ denotes the wing, Wl the winlets, F the fuselage, VTP the Vertical Tail Piece and HTP the Horizontal Tail Piece. $\mathrm{B}$ denotes horizontal bending, $\mathrm{T}$ torsion, FAB denotes fore-after bending, LB lateral bending, BL bending left, BR bending right and VB vertical bending.

\begin{tabular}{llllllll}
\hline No & Mode & $f_{F E, \text { init }}$ & $\mu_{f}(\mathrm{~Hz})$ & $\sigma_{f}(\mathrm{~Hz})$ & $\underline{f}(\mathrm{~Hz})$ & $\bar{f}(\mathrm{~Hz})$ & nsam \\
\hline 1 & RB-Yaw & - & 0.23 & 0.006 & 0.22 & 0.24 & 41 \\
2 & RB-Roll & 0.56 & 0.65 & 0.019 & 0.6 & 0.68 & 81 \\
3 & RB-Pitch & 0.82 & 0.83 & 0.017 & 0.8 & 0.88 & 83 \\
4 & RB-Heave & 2.14 & 2.17 & 0.024 & 2.11 & 2.22 & 86 \\
5 & $2^{\text {nd }}$ W-B & 5.65 & 5.5 & 0.004 & 5.49 & 5.52 & 86 \\
6 & $3^{\text {rd }}$ W-B & 15.11 & 14.91 & 0.017 & 14.88 & 14.94 & 86 \\
7 & $1^{\text {st }}$ assym. W-T & 31.31 & 31.96 & 0.02 & 31.92 & 32.01 & 86 \\
8 & $1^{\text {st }}$ sym. W-T & 33.62 & 32.33 & 0.017 & 32.29 & 32.38 & 86 \\
9 & $1^{\text {st }}$ VTP-B & 35.39 & 34.38 & 0.081 & 34.23 & 34.54 & 86 \\
10 & $4^{\text {th }}$ W-B & 44.66 & 43.89 & 0.015 & 43.85 & 43.92 & 86 \\
11 & $1^{\text {st }}$ W-FAB & 47.21 & 46.71 & 0.149 & 46.27 & 46.99 & 86 \\
12 & $2^{\text {nd }}$ W-FAB & 52.91 & 51.88 & 0.012 & 51.84 & 51.91 & 86 \\
13 & $5^{\text {th }}$ W-B & 60.59 & 58.59 & 0.075 & 58.33 & 58.76 & 86 \\
14 & $1^{\text {st }}$ VTP-T & 67.69 & 65.93 & 0.274 & 65.46 & 66.33 & 86 \\
15 & $2^{\text {nd }}$ F-LB & 102.59 & 100.05 & 0.28 & 99.38 & 100.48 & 86 \\
16 & $2^{\text {nd }}$ VTP-B & 128.62 & 124.56 & 0.356 & 123.85 & 125.1 & 86 \\
17 & $6^{\text {th }}$ W-B & 132.08 & 129.38 & 0.107 & 129.12 & 129.66 & 86 \\
18 & $7^{\text {th }}$ W-B & 145.91 & 141.47 & 0.347 & 140.79 & 142.76 & 85 \\
19 & $2^{\text {nd }}$ HTP-B & 206.73 & 205.59 & 1.023 & 203.24 & 206.87 & 86 \\
20 & $1^{\text {st }}$ HTP-FAB & 225.73 & 219.07 & 1.663 & 216.29 & 221.3 & 86 \\
21 & $1^{\text {st }}$ W-BR & 261.53 & 254.73 & 0.557 & 253.41 & 256.48 & 70 \\
22 & $1^{\text {st }}$ W-BL & 262.64 & 255.02 & 0.575 & 253.84 & 256.27 & 81 \\
23 & $3^{\text {rd }}$ W-FAB & 278.71 & 272.08 & 0.374 & 271.17 & 272.89 & 86 \\
24 & $1^{\text {st }}$ Wl-BL & 320.15 & 303.96 & 1.115 & 301.52 & 306.5 & 82 \\
25 & $1^{\text {st }}$ Wl-BR & 321.64 & 304.32 & 2.138 & 301.17 & 310.27 & 83 \\
26 & $3^{\text {rd }}$ F-LB & 324.12 & 313.68 & 1.218 & 309.38 & 314.76 & 86 \\
27 & $2^{\text {nd }}$ sym. W-T & 336.31 & 328.55 & 0.448 & 327.03 & 330.21 & 85 \\
28 & $2^{\text {nd }}$ assym. W-T & 341.15 & 331.18 & 0.528 & 329.64 & 332.95 & 86 \\
29 & $4^{\text {th }}$ W-FAB & 343.55 & 336.21 & 0.647 & 330.88 & 337.41 & 86 \\
30 & $2 n d$ F-VB & 359.54 & 348.68 & 1.141 & 346.75 & 350.3 & 86 \\
\hline & & & & & & & \\
\hline
\end{tabular}




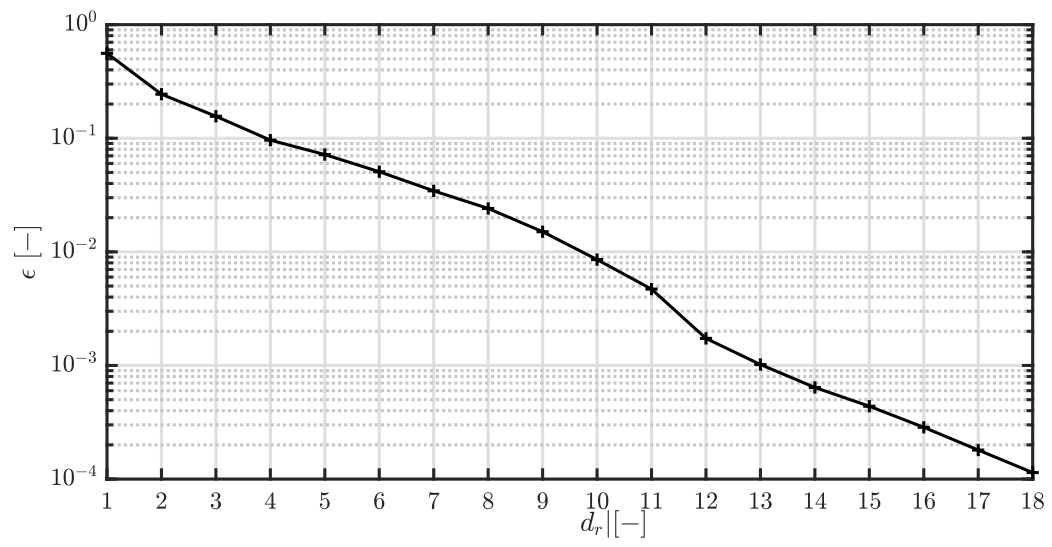

Figure 4: Reduction error $\epsilon$ as a function of the reduced dimension $d_{r}$, applied to the AIRMOD data set.

to compute $\left(\begin{array}{c}d_{r} \\ 2\end{array}\right)$ 2-dimensional convex hulls. The computations are made using the measurement data set with the 14 pre-selected Eigenmodes, containing a selection of either 25, 50 or 86 replica (i.e., the full dataset). All computations are made using a single-thread of an Intel Xeon E5-1620 @ $3.70 \mathrm{GHz}$ with a total $32 \mathrm{~Gb}$ of RAM as to prevent swapping. As can be seen, the projection method is very effective in reducing the computational burden of obtaining the convex hull. Specifically, when considering a 14-dimensional convex hull, the computational cost is reduced with a factor up to $1 \cdot 10^{05}$ when compared to the computation of the full-dimensional convex hull for $d_{r}=14$. Finally, the influence of the measurement data size is considerably smaller when $d_{r}^{+}=2$, which is very relevant for reducing the result of the interval FE model, which contains $2^{16}=65536$ responses.

Note that this does not limit the accuracy of the method since quadratic and higher order interactions between responses are already linearised by considering only the convex hull of the set. All interval quantification computations are therefore performed using 91 2-dimensional projections of the full 14-dimensional convex hull, where an equal weight is attributed to each projection. 


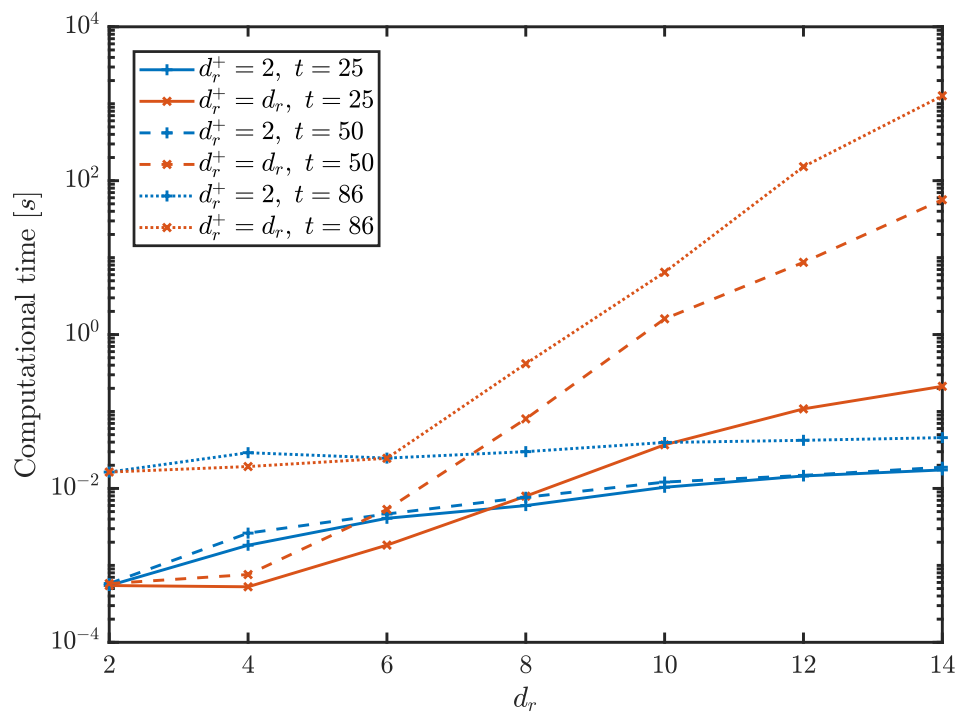

Figure 5: Wall-clock time that is needed to compute $1 d_{r}$-dimensional convex hull, as well as the time needed to compute $\left(\begin{array}{l}d_{r} \\ d_{r}^{+}\end{array}\right) d_{r}^{+}$dimensional convex hulls

\subsubsection{Uncertainty Quantification}

The objective function presented in eq. 12 is in general not convex and should be solved in 32-dimensional space. Therefore, eq. [12 is solved using a hybrid Particle Swarm Algorithm (PSA). A swarm size of 100 particles is used, and the optimization is considered to be converged when it reached 15 stalling iterations. These settings are found in a heuristic approach and based on prior experience with PSA. In a second step a sequential quadratic programming approach is followed until converged, starting from the estimate of the global minimum of eq. 12 obtained by the PSA. The optimality of this solution can be verified by qualitatively comparing cross-sections of the convex hulls of the measurement data set and the result of the interval FE model. For the quantification of the multivariate interval uncertainty the weighting factor $w_{o}$ in eq. 12 is set to unity, meaning that equal priority is given to maximising the overlap and matching the multidimensional volumes. Given the already challenging nature of the optimisation, this weight value prevents the encounter 
of instabilities. These would inevitably appear in the case a large $w_{o}$ is selected due to the then discontinuities behaviour of eq. 12 as a result of the barrierfunction like influence term $w_{o} \Delta V_{o, i}^{2}$.

\subsection{Bayesian model updating}

The Bayesian uncertainty quantification was performed using 18 uncorrelated marginal uniform prior distributions. The range of the distribution of each parameter has been selected spanning an interval from $5 \%$ to $200 \%$ of the parameter nominal value. The likelihood, as introduced in eq. 16), is constructed under the assumption independence of the data.

\subsection{UQ with full data set}

Figure 6 illustrates the marginal posterior distributions of the model parameters obtained via Bayesian updating, and the corresponding quantified interval bounds. For the sake of comparison, the parameters $\boldsymbol{\theta}$ are here normalised by their initial nominal values. The colour coding represents the normalised height of the histogram of each normalised $\theta_{i}$ value according to the obtained marginal posterior. On the other hand, the upward and downward facing triangles denote respectively the lower and upper interval bounds, as obtained by the multivariate interval quantification method. The correlation matrix of the Bayesian result is illustrated in figure 7 .

First, a large conceptual difference between both methods can be noted. On the one hand, the intervals provide the analyst with crisp bounds for the possible parameter values, on the other hand, the marginal posterior distributions assign a non-zero plausibility to a large range of responses. Note that the range of illustrated values is limited due to the finite data set that is used to plot the normalised histograms. Nevertheless, the most probable point in the Bayesian analysis is still clearly identifiable in most cases. It should be noted that the Bayesian analysis predicts a multi-modal plausibility distribution for $\theta_{1}, \theta_{2}$,

$\theta_{4}, \theta_{5}, \theta_{7}, \theta_{8}, \theta_{9}, \theta_{10}, \theta_{12}$ and $\theta_{15}$. The multi-modality can be interpreted as slight changes in the test conditions that were not noted during the experimental 


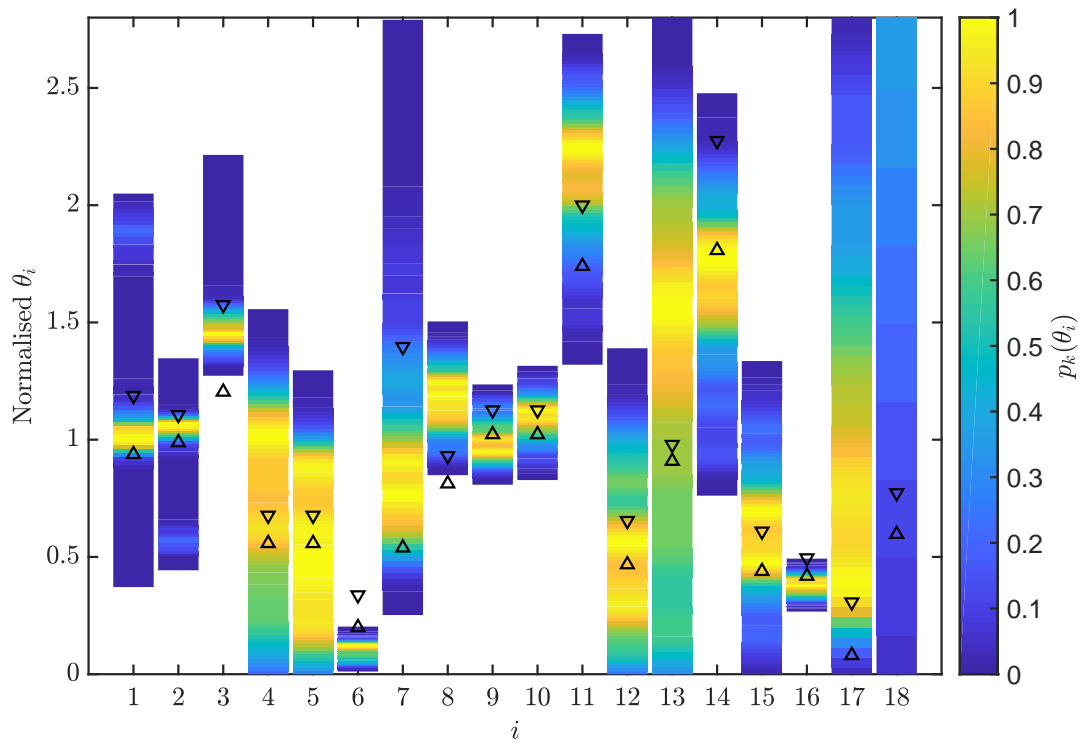

Figure 6: Normalised histograms of the posterior distribution samples, indicated as colour coded bar graphs, and interval bounds, indicated as triangles that are obtained by performing respectively Bayesian inference and Multivariate interval quantification on the full AIRMOD data set.

campaign (see e.g. 33]). This multi-modal behaviour cannot be captured by the interval UQ. By comparing the results for those parameters with multi-modal posteriors, the interval analysis either encompasses all modal peaks (e.g., $\left.\theta_{7}\right)$, or just the most probable one (e.g., $\theta_{12}$ or $\theta_{15}$ ).

Qualitatively, the predictions of both methods correspond rather well, as the interval method usually bounds the same area in parameter space to which the Bayesian method assigns the highest degree of plausibility. Exceptions hereto exists for $\theta_{6}, \theta_{8}, \theta_{16}, \theta_{17}$ and $\theta_{18}$. These differences are due to a variety of factors, such as the difficulty in finding the exact global minimum in a 18dimensional uncertain space in case of the interval method, a highly peaked marginal distributions in the case of Bayesian UQ, or a low-importance input that cannot be effectively updated with the available experiments. As a last remark, the assumption that parameters $4-5$ and $9-10$ are dependent to 
reduce the number of uncertain parameters in the case of the interval analysis is proven to be reasonable, as the marginal posteriors obtained by Bayesian UQ show similar plausibility distributions and they were found to be highly correlated.

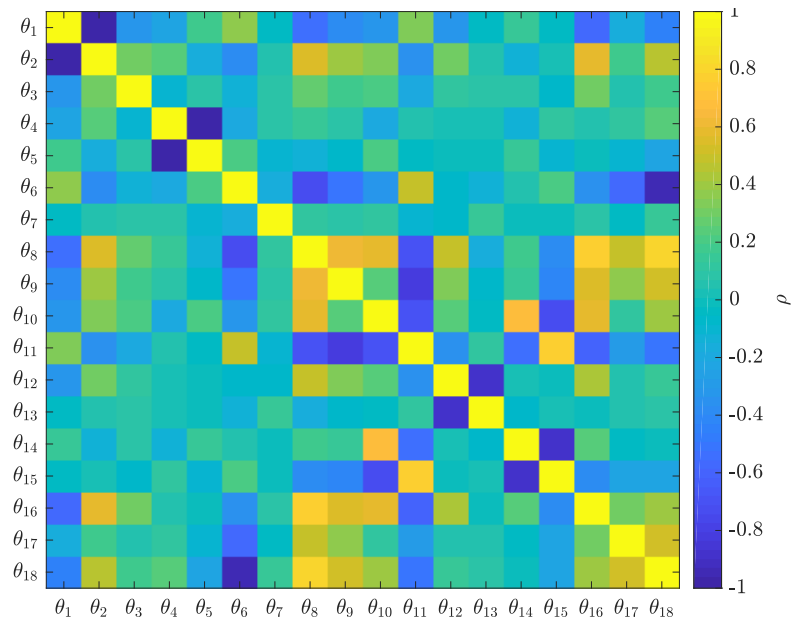

Figure 7: Correlation matrix, obtained by the Bayesian analysis on the full dataset.

From the point of view of the updated model outputs, Figure 8 shows all combinations of the pre-selected eigenfrequencies, obtained by propagating the quantified intervals and Bayesian posterior distribution through their corresponding ANN surrogates of the AIRMOD FE model. As an additional comparison, the measurement data set is also shown.

Marginally, the results obtained by the multivariate interval quantification method predict a wider range of possible responses for $f_{5}, f_{7}, f_{8}, f_{10}$ and $f_{12}$ as compared to the samples from the Bayesian posterior distribution. Note that these bounds, as opposed to the ones predicted by these Bayesian samples, are considered strict. For e.g., $f_{19}$ and $f_{20}$, it is shown that some of the experimental responses are not encapsulated within the convex hull. This is a direct result of the construction of the objective function for the quantification, as elaborated in the final paragraph of section 2.2 . This effect is clearly visible in this case since $w_{o}=1$ to smooth the objective function. 
As concerns the combination of the 5-8, 7-8 and 8-12 eigenmodes, the mutual dependence is not predicted correctly by the quantified interval model. The interval model in fact predicts these responses to be highly dependent, whereas the measurement data show otherwise. Keeping in mind that the $7^{\text {th }}, 8^{\text {th }}$ and $12^{\text {th }}$ eigenmode correspond to respectively anti-symmetric torsion, symmetric torsion and wing fore-after bending modes, the inaccurate prediction of their dependence in the interval model is a direct cause of the assumption that $\theta_{4}$ $\theta_{5}$ and $\theta_{9}-\theta_{10}$ are fully dependent.

Finally, although the Bayesian posterior distributions predict a non-zero plausibility for a larger range of parameter values as compared to the interval model, tighter bounds on the prediction of the uncertain parameters as compared to the measurement data set are obtained. This observation stems from the low plausibility that is attributed to the tails of the marginal posterior distributions, combined with the peakedness of the distribution, such that the moderately small dataset of 500 samples did not sample these tails.

These results indicate that, in case sufficient data are available, Bayesian inference outperforms the multivariate quantification method in terms of information content (e.g., correlation and multi-modality as compared to purely the crisp bounds) and accuracy (less over-conservative while still capturing almost 480 all responses). Note that a part of the discrepancy between the results obtained by both methods stems from different assumptions on parameter dependence that were made. This however does not invalidate this main conclusion.

\subsection{UQ with small data set}

Figure 9 shows all combinations of considered eigenfrequencies, obtained by propagating the quantified intervals and posterior distributions through the AIRMOD FE model.

Concerning the marginal eigenfrequencies, the bounds predicted by the quantified interval method circumscribe the measurement data set tighter as compared to the Bayesian samples, which are shown to be more over-conservative. This is e.g., the case for $f_{1}, f_{2}, f_{3}, f_{4}, f_{5}, f_{11}, f_{14}$ and $f_{19}$. The only excep- 


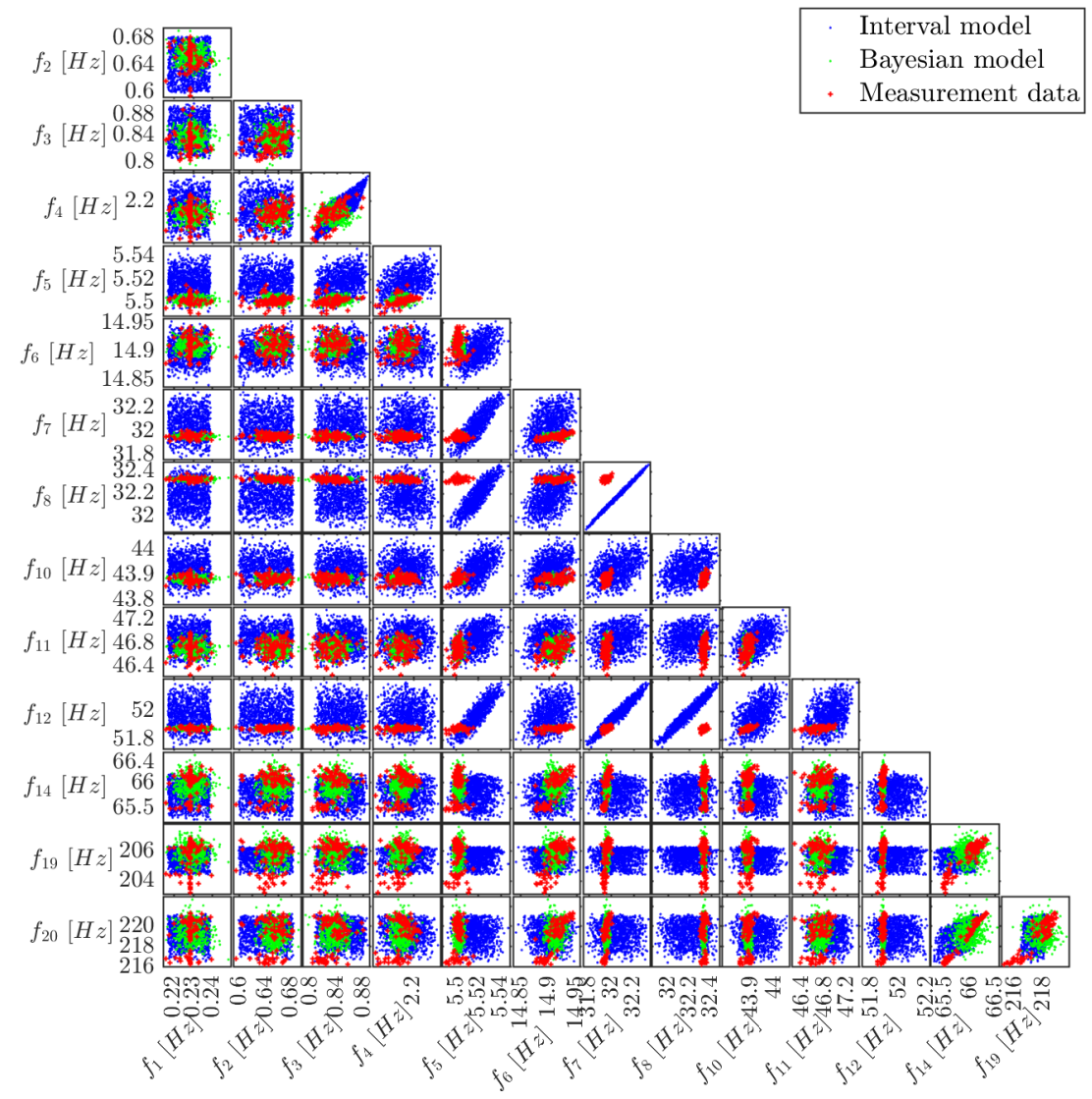

Figure 8: All combinations of considered eigenfrequencies, obtained by propagating the quantified intervals and posterior distributions through the AIRMOD FE model. The quantified results were obtained by using the full measurement data set.

tion hereto is the $8^{\text {th }}$ eigenmode (i.e. the $1^{\text {st }}$ symmetric wing torsion mode), which is completely missed by the quantified interval method, as it focussed on encompassing $f_{7}$ since both frequencies are equal as can be noted from figure 9. The main reason for this lack of overlap is explained by the assumed perfect dependence between parameters $\theta_{4}-\theta_{5}$ and $\theta_{9}-\theta_{10}$, combined with the low data availability. Ideally, the interval method would try to find a set of intervals that encompass both $f_{7}$ and $f_{8}$ perfectly, but due to the fact that $w_{0}$ is set to unity, this did not happen. This is a possible limitation of the method. 
Due to the scarcity of the dataset, none of the methods was able to give an accurate estimate of the dependence between the eigenfrequencies. However, the interval method, apart from the $8^{\text {th }}$ eigenmode, was able to provide tighter bounds on the uncertainty. The ability to provide a better estimate is caused by the effect of the prior distribution for the Bayesian UQ on the identified posterior. To recall, a uniform distribution with very broad basis was selected as to correspond with no prior knowledge on the uncertainty. Since insufficient data are available to compensate for the effect of this prior, the Bayesian method provides a very broad estimate of possible responses.

Therefore, under scarce data, the interval method outperforms the Bayesian method since the lack of informative data makes the quantified posterior highly dependent on the prior knowledge. The interval method on the other hand does not need an initial estimate of the parameter uncertainty, since the global optimisation routine actively searches the space of input parameters for those intervals that best prescribe the available data.

\section{Conclusions}

In this paper, an improved version of a novel interval quantification method was presented in the context of high-dimensional models, which has been shown to be able to alleviate the curse of dimensionality that is attributed to the necessary convex hull computations. The latter is illustrated by the factor $10^{5}$ gain in computational efficiency for the evaluation of the discrepancy between the convex hulls.

The approach has been tested by analysing the DLR-AIRMOD test structure and compared with results obtained by Bayesian model updating. Concerning the interval method with the full dataset, the cross-sections of the resulting convex hull failed to encompass the dataset completely, which is a direct result of some assumptions that were made in the modelling process. Moreover, propagation of the Bayesian posterior distribution provided a tighter estimation of the experimental dataset. The interval method however provides the analyst 


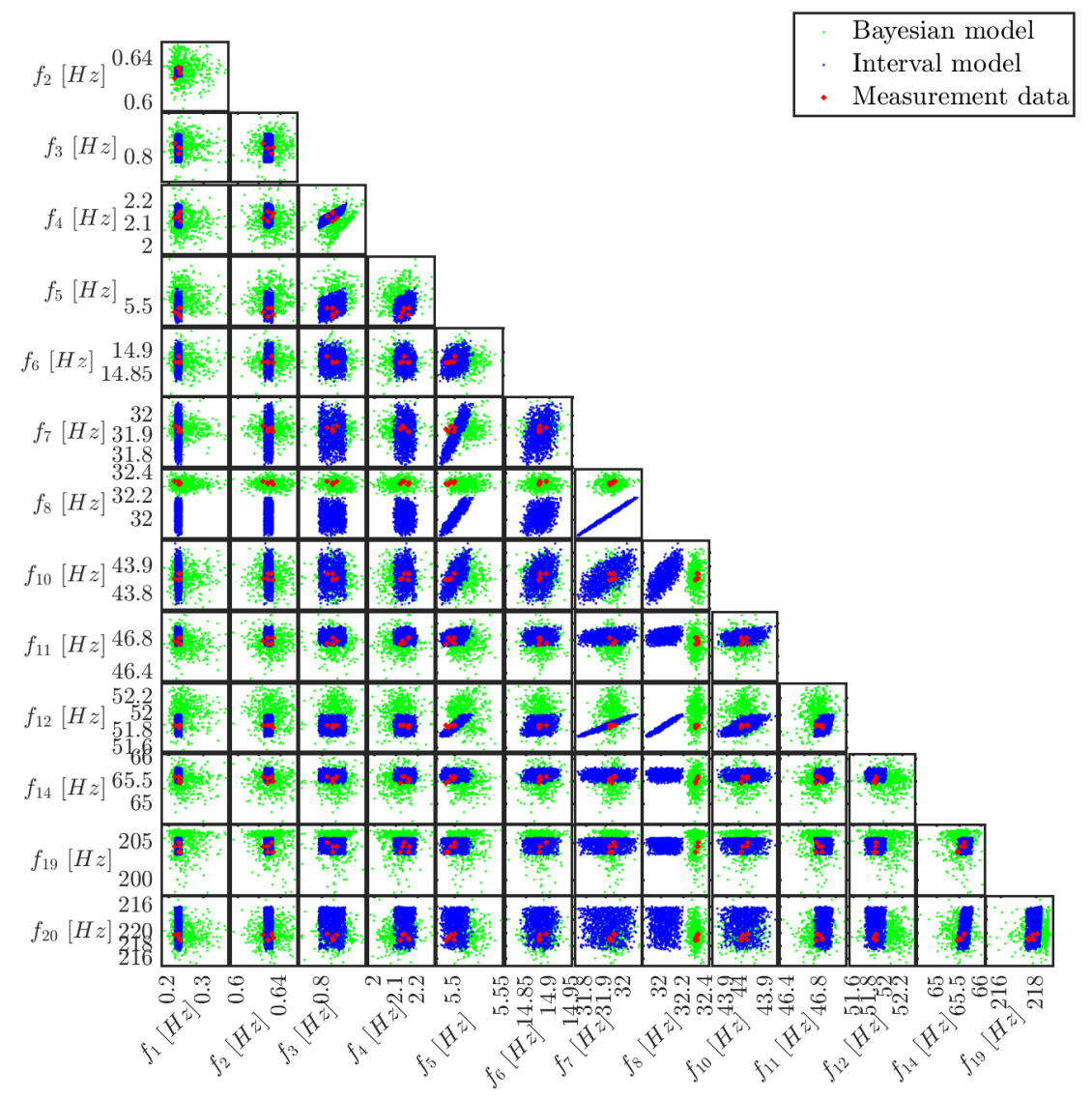

Figure 9: All combinations of considered eigenfrequencies, obtained by propagating the quantified intervals and posterior distributions through the AIRMOD FE model. The quantified results were obtained by using only 5 measured replica.

with fixed bounds between the uncertainty is believed to be located in contrast to the possibly infinite support of the posterior distributions stemming from the Bayesian analysis. In case only limited data are available, the effect of the prior distribution dominates the obtained posterior distribution in the Bayesian case. In this case, propagation of the quantified intervals provides the analyst with a better representation of the measurement dataset in terms of over-conservatism and crispness of the model representation. However, the case study illustrates clearly that it is challenging to perfectly fit a numerically computed convex hull 
to a convex hull over experimental data. This is a direct result of the fact that in the context of inverse interval uncertainty quantification, a trade-off between conservatism and representing the data accurately exists, where some data points are missed to better represent the full dataset. This was specifically evident when only 5 data samples are considered, as one eigenfrequency was missed by the interval method.

As such, using this challenging high-dimensional example and the presented theoretical discussion, it is shown that the choice between interval and Bayesian UQ boils down to some key questions an analyst has to consider:

- what is the desired information on the uncertainty?

If the analyst is only interested in bounds on the uncertain parameters, given a set of measurement data, interval analysis has been shown to be very robust with respect to the scarcity of the measurement data. In this case no prior, often subjective, knowledge is needed for the analysis. Should the analyst on the other hand be interested in a complete description of the (joint-)plausibility, including correlation and multi-modal descriptors, a Bayesian approach has to be applied.

- what is the availability of data?

Since the prior distribution influences the posterior distribution in a Bayesian context under scarce data to a large extent, sufficient informative data should be available to perform a Bayesian analysis. If insufficient data are available, but the analyst requires the information provided by Bayesian analysis; (s)he should collect more data or take utmost care in constructing the prior distribution. Otherwise, the interval approach was proven to give more informative bounds in this case.

\section{Acknowledgements}

The authors would like to acknowledge the financial support of the Flemish Research Foundation (FWO) for travel grants K218117N and K217917N and the research project $\mathrm{G} 0 \mathrm{C} 2218 \mathrm{~N}$. 
[1] S. Ferson, L. R. Ginzburg, Different methods are needed to propagate ignorance and variability, Reliability Engineering \& System Safety 54 (2) (1996) 133 - 144. doi:http://dx.doi.org/10.1016/S0951-8320(96)00071-3.

[2] W. Oberkampf, S. DeLand, B. Rutherford, K. Diegert, K. Alvin, A new methodology for the estimation of total uncertainty in computational simulation, in: A new methodology for the estimation of total uncertainty in computational simulation, 1999, pp. 3061-3083.

[3] G. Stefanou, The stochastic finite element method: Past, present and future, Computer Methods in Applied Mechanics and Engineering 198 (9-12) (2009) 1031-1051. doi:10.1016/j.cma.2008.11.007.

[4] D. Moens, M. Hanss, Non-probabilistic finite element analysis for parametric uncertainty treatment in applied mechanics: Recent advances, Finite Elements in Analysis and Design 47 (1) (2011) 4-16. doi:10.1016/j. finel.2010.07.010

[5] M. Beer, S. Ferson, V. Kreinovich, Imprecise probabilities in engineering analyses, Mechanical systems and signal processing 37 (1) (2013) 4-29.

[6] J. L. Beck, L. S. Katafygiotis, Updating models and their uncertainties. i: Bayesian statistical framework, Journal of Engineering Mechanics 124 (4) (1998) 455-461.

585

[7] L. Mehrez, A. Doostan, D. Moens, D. Vandepitte, Stochastic identification of composite material properties from limited experimental databases, Part II: Uncertainty modelling, Mechanical Systems and Signal Processing 27 (2012) 484-498. doi:10.1016/j.ymssp.2011.09.001.

[8] F. Fedele, R. L. Muhanna, N. Xiao, R. L. Mullen, Interval-Based Approach 590 for Uncertainty Propagation in Inverse Problems, Journal of Engineering Mechanics 4 (1) (2014) 1-7. doi:10.1061/(ASCE) EM.1943-7889.0000815 
[9] Z. Deng, Z. Guo, X. Zhang, Interval model updating using perturbation method and radial basis function neural networks, Mechanical Systems and Signal Processing 84 (2017) 699-716.

[10] J. Mottershead, M. Friswell, Model updating in structural dynamics: a survey, Journal of sound and vibration 167 (2) (1993) 347-375.

[11] H. H. Khodaparast, J. E. Mottershead, K. J. Badcock, Interval model updating with irreducible uncertainty using the Kriging predictor, Mechanical Systems and Signal Processing 25 (4) (2011) 1204-1206. doi: 10.1016/j.ymssp.2010.10.009.

[12] Y. Govers, H. H. Khodaparast, M. Link, J. Mottershead, A comparison of two stochastic model updating methods using the $\{\mathrm{DLR}\}\{$ AIRMOD test structure, Mechanical Systems and Signal Processing 5253 (2015) 105 -114. doi:https://doi.org/10.1016/j.ymssp.2014.06.003

[13] M. Faes, J. Cerneels, D. Vandepitte, D. Moens, Identification and quantification of multivariate interval uncertainty in finite element models, Computer Methods in Applied Mechanics and Engineering 315 (2017) 896 920. doi:http://dx.doi.org/10.1016/j.cma.2016.11.023.

[14] M. Faes, D. Moens, Identification and quantification of spatial interval uncertainty in numerical models, Computers \& Structures 192 (2017) 1633.

[15] M. Faes, D. Moens, Quantification of non-homogeneous interval uncertainty based on scatter in modal properties, Procedia Engineering 199 (2017) $1216-1221$.

[16] I. T. Jolliffe, J. Cadima, Principal component analysis: a review and recent developments, Phil. Trans. R. Soc. A 374 (2065) (2016) 20150202.

[17] L. Van Der Maaten, Accelerating t-sne using tree-based algorithms., Journal of machine learning research 15 (1) (2014) 3221-3245. 
[18] P. G. Constantine, E. Dow, Q. Wang, Active subspace methods in theory and practice: applications to kriging surfaces, SIAM Journal on Scientific Computing 36 (4) (2014) A1500-A1524.

[19] D. Moens, D. Vandepitte, Recent advances in non-probabilistic approaches for non-deterministic dynamic finite element analysis, Archives of Com-

【 putational Methods in Engineering 13 (3) (2006) 389-464. doi:10.1007/ BF02736398.

[20] M. Beer, V. Kreinovich, Interval or moments: Which carry more in-

घ formation?, Soft Computing 17 (8) (2013) 1319-1327. doi:10.1007/ s00500-013-1002-1.

[21] L. S. Katafygiotis, J. L. Beck, Updating models and their uncertainties. ii:model identifiability, Journal of Engineering Mechanics 124 (4) (1998) 463-467.

[22] Y. Govers, H. Khodaparast, M. Link, J. Mottershead, Stochastic Model Updating of the DLR AIRMOD Structure, in: Vulnerability, Uncertainty,

(4) and Risk (CASCE 2014, no. 1974, Liverpool, 2014, pp. 475-484. doi: $10.1061 / 9780784413609.049$

[23] V. Kreinovich, J. Beck, C. Ferregut, A. Sanchez, G. R. Keller, M. Averill, S. A. Starks, Monte-carlo-type techniques for processing interval uncertainty, and their potential engineering applications, Reliable Computing 13 (1) (2007) 25-69.

[24] M. Hanss, The transformation method for the simulation and analysis of systems with uncertain parameters, Fuzzy Sets and Systems 130 (3) (2002) 277-289. doi:10.1016/S0165-0114(02)00045-3.

[25] C. B. Barber, D. P. Dobkin, H. Huhdanpaa, The quickhull algorithm for convex hulls, ACM Transactions on Mathematical Software 22 (4) (1996) 469-483. doi:10.1145/235815.235821. 
[26] B. Schölkopf, A. Smola, K.-R. Müller, Nonlinear component analysis as a kernel eigenvalue problem, Neural Comput. 10 (5) (1998) 1299-1319.

[27] J. B. Tenenbaum, V. De Silva, J. C. Langford, A global geometric framework for nonlinear dimensionality reduction, Science 290 (5500) (2000) $2319-2323$.

[28] S. T. Roweis, L. K. Saul, Nonlinear dimensionality reduction by locally linear embedding, Science 290 (5500) (2000) 2323-2326.

[29] R. Rocchetta, M. Broggi, Q. Huchet, E. Patelli, On-line bayesian model updating for structural health monitoring, Mechanical Systems and Signal Processing 103 (2018) 174-195.

[30] J. Ching, Y.-C. Chen, Transitional markov chain monte carlo method for bayesian model updating, model class selection, and model averaging, Journal of engineering mechanics 133 (7) (2007) 816-832.

[31] M. Tian, D.-Q. Li, Z.-J. Cao, K.-K. Phoon, Y. Wang, Bayesian identification of random field model using indirect test data, Engineering Geology 210 (2016) 197-211.

[32] M. Stein, M. Beer, V. Kreinovich, Bayesian approach for inconsistent information, Information sciences 245 (2013) 96-111.

[33] E. Patelli, Y. Govers, M. Broggi, H. M. Gomes, M. Link, J. E. Mottershead, Sensitivity or bayesian model updating: a comparison of techniques using

1 the dlr airmod test data, Archive of Applied Mechanics (2017) 1-21doi: $10.1007 / \mathrm{s} 00419-017-1233-1$.

[34] E. Patelli, M. Broggi, Y. Govers, J. E. Mottershead, Model updating strategy of the dlr-airmod test structure, Procedia Engineering 199 (2017) 978983.

[35] S. Adhikari, Rates of change of eigenvalues and eigenvectors in damped dynamic system, AIAA Journal 37 (11) (1999) 1452-1458. doi:10.2514/ 3.14342 